\title{
Fock Spaces for the q-Dunkl Kernel
}

\author{
Fethi Soltani* \\ Higher College of Technology and Informatics, Tunis, Tunisia \\ Email: fethisoltani10@yahoo.com
}

Received November 26, 2011; revised January 4, 2012; accepted January 20, 2012

\begin{abstract}
In this work, we introduce a class of Hilbert spaces $\mathcal{F}_{q, \alpha}$ of entire functions on the disk $D\left(0, \frac{1}{1-q}\right), 0<q<1$, with reproducing kernel given by the $q$-Dunkl kernel $E_{\alpha}\left(z ; q^{2}\right)$. The definition and properties of the space $\mathcal{F}_{q, \alpha}$ extend naturally those of the well-known classical Fock space. Next, we study the multiplication operator $Q$ by $z$ and the $q$-Dunkl operator $\Lambda_{q, \alpha}$ on the Fock space $\mathcal{F}_{q, \alpha}$; and we prove that these operators are adjoint-operators and continuous from this space into itself.
\end{abstract}

Keywords: Generalized $q$-Fock Spaces; $q$-Dunkl Kernel; $q$-Dunkl Operator; $q$-Translation Operators

\section{Introduction}

Fock space $\mathcal{F}$ (called also Segal-Bargmann space [1]) is the Hilbert space of entire functions

$$
\begin{aligned}
f(z)=\sum_{n=0}^{\infty} a_{n} z^{n} \text { on } \mathbb{C} \text { such that } \\
\|f\|_{\mathcal{F}}^{2}:=\sum_{n=0}^{\infty}\left|a_{n}\right|^{2} n !<\infty .
\end{aligned}
$$

This space was introduced by Bargmann in [2] and it was the aim of many works [1]. Especially, the differential operator $D=\mathrm{d} / \mathrm{d} z$ and the multiplication operator by $z$ are densely defined, closed and adjoint-operators on $\mathcal{F}$ (see [2]).

In [3], Sifi and Soltani introduced a Hilbert space $\mathcal{F}_{\alpha}$ of entire functions on $\mathbb{C}$, where the inner product is weighted by the modified Macdonald function. On $\mathcal{F}_{\alpha}$ the Dunkl operator

$$
\begin{aligned}
& \Lambda_{\alpha} f(z):=\frac{\mathrm{d}}{\mathrm{d} z} f(z)+\frac{2 \alpha+1}{z}\left[\frac{f(z)-f(-z)}{2}\right], \\
& \alpha>-1 / 2,
\end{aligned}
$$

and the multiplication by $z$ are densely defined, closed and adjoint-operators.

In this paper, we consider the $q$-Dunkl kernel:

$$
E_{\alpha}\left(x ; q^{2}\right):=\sum_{n=0}^{\infty} \frac{x^{n}}{b_{n}\left(\alpha ; q^{2}\right)},
$$

where $b_{n}\left(\alpha ; q^{2}\right)$ are given later in Section 2. We dis-

\footnotetext{
*Author partially supported by DGRST project 04/UR/15-02 and CMCU program 10G 1503.
}

cuss some properties of a class of Fock spaces associated to the $q$-Dunkl kernel and we give some applications.

In this work, building on the ideas of Bargmann and Cholewinski [4], we define the $q$-Fock space $\mathcal{F}_{q, \alpha}$ as the space of entire functions $f(z)=\sum_{n=0}^{\infty} a_{n} z^{n}$ on the disk $D\left(0, \frac{1}{1-q}\right)$ of center 0 and radius $\frac{1}{1-q}$, and such that

$$
\|f\|_{\mathcal{F}_{q, \alpha}}^{2}:=\sum_{n=0}^{\infty}\left|a_{n}\right|^{2} b_{n}\left(\alpha ; q^{2}\right)<\infty .
$$

Let $f$ and $g$ be in $\mathcal{F}_{q, \alpha}$, such that $f(z)=\sum_{n=0}^{\infty} a_{n} z^{n}$ and $g(z)=\sum_{n=0}^{\infty} c_{n} z^{n}$, the inner product is given by

$$
\langle f, g\rangle_{\mathcal{F}_{q, \alpha}}=\sum_{n=0}^{\infty} a_{n} \bar{c}_{n} b_{n}\left(\alpha ; q^{2}\right) \text {. }
$$

The $q$-Fock space $\mathcal{F}_{q, \alpha}$ has also a reproducing kernel $\mathcal{K}_{q, \alpha}$ given by

$$
\mathcal{K}_{q, \alpha}(w, z)=E_{\alpha}\left(\bar{w} z ; q^{2}\right) ; \quad w, z \in D\left(0, \frac{1}{1-q}\right) .
$$

Then if $f \in \mathcal{F}_{q, \alpha}$, we have

$$
\left\langle f, \mathcal{K}_{q, \alpha}(w, .)\right\rangle_{\mathcal{F}_{q, \alpha}}=f(w), \quad w \in D\left(0, \frac{1}{1-q}\right) .
$$

Using this property, we prove that the space $\mathcal{F}_{q, \alpha}$ is a Hilbert space and we give an Hilbert basis.

Next, using the previous results, we consider the multiplication operator $Q$ by $z$ and the $q$-Dunkl operator $\Lambda_{q, \alpha}$ on the Fock space $\mathcal{F}_{q, \alpha}$, and we prove that these 
operators are continuous from $\mathcal{F}_{q, \alpha}$ into itself, and satisfy:

$$
\begin{gathered}
\left\|\Lambda_{q, \alpha} f\right\|_{\mathcal{F}_{q, \alpha}} \leq C_{q, \alpha}|f|_{\mathcal{F}_{q, \alpha}}, \\
\|Q f\|_{\mathcal{F}_{q, \alpha}} \leq C_{q, \alpha}|f|_{\mathcal{F}_{q, \alpha}},
\end{gathered}
$$

where $C_{q, \alpha}$ is a constant independent of $f$.

Then, we prove that these operators are adjoint-operators on $\mathcal{F}_{q, \alpha}$ :

$$
\langle Q f, g\rangle_{\mathcal{F}_{q, \alpha}}=\left\langle f, \Lambda_{q, \alpha} g\right\rangle_{\mathcal{F}_{q, \alpha}} ; f, g \in \mathcal{F}_{q, \alpha} .
$$

Lastly, we define and study on the Fock space $\mathcal{F}_{q, \alpha}$, the $q$-translation operators:

$$
T_{z} f(w):=E_{\alpha}\left(z \Lambda_{q, \alpha} ; q^{2}\right) f(w) ; \quad w, z \in D\left(0, \frac{1}{1-q}\right),
$$

and the generalized multiplication operators:

$$
M_{z} f(w):=E_{\alpha}\left(z Q ; q^{2}\right) f(w) ; \quad w, z \in D\left(0, \frac{1}{1-q}\right) .
$$

Using the continuous properties of $\Lambda_{q, \alpha}$ and $Q$ we deduce also that the operators $T_{z}$ and $M_{z}$, for $|z|<\frac{1}{(1-q)\left(2-q^{2 \alpha+1}\right)}$, are continuous from $\mathcal{F}_{q, \alpha}$ into itself, and satisfy:

$$
\begin{aligned}
& \left\|T_{z} f\right\|_{\mathcal{F}_{\mathcal{q}, \alpha}} \leq E_{\alpha}\left(C_{q, \alpha}|z| ; q^{2}\right)\|f\|_{\mathcal{F}_{q, \alpha}}, \\
& \left\|M_{z} f\right\|_{\mathcal{F}_{q, \alpha}} \leq E_{\alpha}\left(C_{q, \alpha}|z| ; q^{2}\right)\|f\|_{\mathcal{F}_{q, \alpha}} .
\end{aligned}
$$

\section{Preliminaries and the $q$-Fock Spaces $\mathcal{F}_{q, \alpha}$}

Let $a$ and $q$ be real numbers such that $0<q<1$; the $q$-shifted factorial are defined by

$$
(a ; q)_{0}:=1, \quad(a ; q)_{n}:=\prod_{i=0}^{n-1}\left(1-a q^{i}\right), \quad n=1,2, \cdots, \infty .
$$

Jackson [5] defined the $q$-analogue of the Gamma function as

$$
\Gamma_{q}(x):=\frac{(q ; q)_{\infty}}{\left(q^{x} ; q\right)_{\infty}}(1-q)^{1-x}, \quad x \neq 0,-1,-2, \cdots
$$

It satisfies the functional equation

$$
\Gamma_{q}(x+1)=\frac{1-q^{x}}{1-q} \Gamma_{q}(x), \quad \Gamma_{q}(1)=1,
$$

and tends to $\Gamma(x)$ when $q$ tends to $1^{-}$. In particular, for $n=1,2, \cdots$, we have

$$
\Gamma_{q}(n+1)=\frac{(q ; q)_{n}}{(1-q)^{n}} .
$$

The $q$-derivative $D_{q} f$ of a suitable function $f$ (see [6]) is given by

$$
D_{q} f(x):=\frac{f(x)-f(q x)}{(1-q) x}, \quad x \neq 0,
$$

and $D_{q} f(0)=f^{\prime}(0)$ provided $f^{\prime}(0)$ exists.

If $f$ is differentiable then $D_{q} f(x)$ tends to $f^{\prime}(x)$ as $q \rightarrow 1^{-}$.

Taking account of the paper [3] and the same way, we define the $q$-Dunkl kernel by

$$
E_{\alpha}\left(x ; q^{2}\right):=I_{\alpha}\left(x ; q^{2}\right)+\frac{x}{(1+q)[\alpha+1]_{q^{2}}} I_{\alpha+1}\left(x ; q^{2}\right),
$$

where $I_{\alpha}\left(x ; q^{2}\right)$ is the $q$-modified Bessel function $[7,8]$ given by

$$
\begin{aligned}
& I_{\alpha}\left(x ; q^{2}\right) \\
& :=\Gamma_{q^{2}}(\alpha+1) \sum_{n=0}^{\infty} \frac{x^{2 n}}{(1+q)^{2 n} \Gamma_{q^{2}}(n+1) \Gamma_{q^{2}}(n+\alpha+1)} .
\end{aligned}
$$

Furthermore, the Dunkl kernel $E_{\alpha}\left(x ; q^{2}\right)$ can be expanded in a power series in the form

$$
E_{\alpha}\left(x ; q^{2}\right):=\sum_{n=0}^{\infty} \frac{x^{n}}{b_{n}\left(\alpha ; q^{2}\right)},
$$

where

$$
b_{2 n}\left(\alpha ; q^{2}\right):=\frac{(1+q)^{2 n} \Gamma_{q^{2}}(n+1) \Gamma_{q^{2}}(n+\alpha+1)}{\Gamma_{q^{2}}(\alpha+1)},
$$

and

$$
b_{2 n+1}\left(\alpha ; q^{2}\right):=\frac{(1+q)^{2 n+1} \Gamma_{q^{2}}(n+1) \Gamma_{q^{2}}(n+\alpha+2)}{\Gamma_{q^{2}}(\alpha+1)} .
$$

If we put $U_{n}:=\frac{1}{b_{n}\left(\alpha ; q^{2}\right)}$, then

$$
\frac{U_{n}}{U_{n+1}} \rightarrow \frac{1}{(1-q)^{2}}, \quad q \rightarrow 1^{-} .
$$

Thus, the $q$-Dunkl kernel $E_{\alpha}\left(x ; q^{2}\right)$ is defined on $D\left(0, \frac{1}{(1-q)^{2}}\right)$ and tends to the Dunkl kernel $E_{\alpha}(x)$ as $q \rightarrow 1^{-}$.

We consider the $q$-Dunkl operator operator $\Lambda_{q, \alpha}$ defined by

$$
\Lambda_{q, \alpha} f(x):=D_{q} f(x)+\frac{[2 \alpha+1]_{q}}{x}\left[\frac{f(q x)-f(-q x)}{2}\right],
$$

where 


$$
[2 \alpha+1]_{q}:=\frac{1-q^{2 \alpha+1}}{1-q} .
$$

The $q$-Dunkl operator tends to the Dunkl operator $\Lambda_{\alpha}$ as $q \rightarrow 1^{-}$.

Lemma 1. The function $E_{\alpha}\left(\lambda: ; q^{2}\right), \lambda \in D\left(0, \frac{1}{1-q}\right)$, is the unique analytic solution of the q-problem:

$$
\Lambda_{q, \alpha} y(x)=\lambda y(x), \quad y(0)=1 .
$$

Proof. Searching a solution of (2) in the form $y(x)=\sum_{n=0}^{\infty} a_{n} x^{n}$. Then

$$
D_{q} y(x)=\sum_{n=1}^{\infty} a_{n}[n]_{q} x^{n-1} .
$$

Replacing in (2), we obtain

$$
\sum_{n=1}^{\infty} a_{n}\left[[n]_{q}+q^{n}[2 \alpha+1]_{q}\left(\frac{1-(-1)^{n}}{2}\right)\right] x^{n}=\lambda \sum_{n=1}^{\infty} a_{n-1} x^{n} .
$$

Thus,

$$
a_{n}\left[[n]_{q}+q^{n}[2 \alpha+1]_{q}\left(\frac{1-(-1)^{n}}{2}\right)\right]=\lambda a_{n-1}, \quad n=1,2, \cdots .
$$

Using the fact that

$[2 n+1]_{q}+q^{2 n+1}[2 \alpha+1]_{q}=[2 n+2 \alpha+2]_{q}$, we deduce that

$$
a_{2 n}=\frac{\lambda}{[2 n]_{q}} a_{2 n-1} \text { and } a_{2 n+1}=\frac{\lambda}{[2 n+2 \alpha+2]_{q}} a_{2 n} \text {. }
$$

We get

$$
a_{2 n+1}=\frac{\lambda^{2}}{[2 n]_{q}[2 n+2 \alpha+2]_{q}} a_{2 n-1} .
$$

Since $[2 n]_{q}=(1+q)[n]_{q^{2}}$, we deduce

$$
a_{2 n+1}=\frac{\lambda^{2}}{(1+q)^{2}[n]_{q^{2}}[n+\alpha+1]_{q^{2}}} a_{2 n-1} \text {. }
$$

This proves that

$$
a_{2 n+1}=\frac{\lambda^{2 n+1} \Gamma_{q^{2}}(\alpha+1)}{(1+q)^{2 n+1} \Gamma_{q^{2}}(n+1) \Gamma_{q^{2}}(n+\alpha+2)},
$$

and

$$
a_{2 n}=\frac{\lambda^{2 n} \Gamma_{q^{2}}(\alpha+1)}{(1+q)^{2 n} \Gamma_{q^{2}}(n+1) \Gamma_{q^{2}}(n+\alpha+1)} .
$$

Therefore,

$$
\begin{aligned}
& y(x)=\Gamma_{q^{2}}(\alpha+1) \sum_{n=0}^{\infty} \frac{(\lambda x)^{2 n}}{(1+q)^{2 n} \Gamma_{q^{2}}(n+1) \Gamma_{q^{2}}(n+\alpha+1)} \\
& +\Gamma_{q^{2}}(\alpha+1) \sum_{n=0}^{\infty} \frac{(\lambda x)^{2 n+1}}{(1+q)^{2 n+1} \Gamma_{q^{2}}(n+1) \Gamma_{q^{2}}(n+\alpha+2)} \\
& =I_{\alpha}\left(\lambda x ; q^{2}\right)+\frac{\lambda x}{(1+q)[\alpha+1]_{q^{2}}} I_{\alpha+1}\left(\lambda x ; q^{2}\right),
\end{aligned}
$$

which completes the proof of the lemma.

Lemma 2. The constants $b_{n}\left(\alpha ; q^{2}\right), n \in \mathbb{N}$ satisfy the following relations:

1) $b_{n+1}\left(\alpha ; q^{2}\right)$

$$
=\left[[n+1]_{q}+q^{n+1}[2 \alpha+1]_{q}\left(\frac{1+(-1)^{n}}{2}\right)\right] b_{n}\left(\alpha ; q^{2}\right),
$$

2) $b_{2 n+1}\left(\alpha ; q^{2}\right)=(1+q)[\alpha+1]_{q^{2}} b_{2 n}\left(\alpha+1 ; q^{2}\right)$,

3) $b_{n}\left(\alpha ; q^{2}\right)$

$$
:=\frac{(1+q)^{n} \Gamma_{q^{2}}([n / 2]+1) \Gamma_{q^{2}}\left(\left[\frac{n+1}{2}\right]+\alpha+1\right)}{\Gamma_{q^{2}}(\alpha+1)},
$$

where $[n / 2]$ is the integer part of $n / 2$.

Lemma 3. For $k \in \mathbb{N}$, we have

$$
\Lambda_{q, \alpha} z^{k}=\frac{b_{k}\left(\alpha ; q^{2}\right)}{b_{k-1}\left(\alpha ; q^{2}\right)} z^{k-1}, \quad k \geq 1 .
$$

Proof. Since

$$
E_{\alpha}\left(\lambda z ; q^{2}\right):=\sum_{k=0}^{\infty} \frac{(\lambda z)^{k}}{b_{k}\left(\alpha ; q^{2}\right)},
$$

then from Equation (2) we obtain

$$
\sum_{k=1}^{\infty} \frac{\Lambda_{q, \alpha} z^{k}}{b_{k}\left(\alpha ; q^{2}\right)} \lambda^{k}=\sum_{k=1}^{\infty} \frac{z^{k-1}}{b_{k-1}\left(\alpha ; q^{2}\right)} \lambda^{k} .
$$

This clearly yields the result.

Definition 1. Let $\alpha \geq-1 / 2$. The $q$-Fock space $\mathcal{F}_{q, \alpha}$ is the prehilbertian space of entire functions $f(z)=\sum_{n=0}^{\infty} a_{n} z^{n}$ on $D\left(0, \frac{1}{1-q}\right)$, such that

$$
\|f\|_{\mathcal{F}_{q, \alpha}}^{2}:=\sum_{n=0}^{\infty}\left|a_{n}\right|^{2} b_{n}\left(\alpha ; q^{2}\right)<\infty,
$$

where $b_{n}\left(\alpha ; q^{2}\right)$ is given by (1).

The inner product in $\mathcal{F}_{q, \alpha}$ is given for $f(z)=\sum_{n=0}^{\infty} a_{n} z^{n}$ and $g(z)=\sum_{n=0}^{\infty} c_{n} z^{n}$ by 


$$
\langle f, g\rangle_{\mathcal{F}_{q, \alpha}}=\sum_{n=0}^{\infty} a_{n} \overline{c_{n}} b_{n}\left(\alpha ; q^{2}\right) .
$$

Remark 1. If $q \rightarrow 1^{-}$, the space $\mathcal{F}_{q, \alpha}$ agrees with the generalized Fock space associated to the Dunkl operator (see [3]).

Proposition 1. For $f, g \in \mathcal{F}_{q, \alpha}$, we have

$$
\langle f, g\rangle_{\mathcal{F}_{q, \alpha}}=f\left(\Lambda_{q, \alpha}\right) \tilde{g}(0), \quad \tilde{g}(z)=\overline{g(\bar{z})} .
$$

Proof. Given $f(z)=\sum_{n=0}^{\infty} a_{n} z^{n} \in \mathcal{F}_{q, \alpha}$ and $g(z)=\sum_{n=0}^{\infty} c_{n} z^{n} \in \mathcal{F}_{q, \alpha}$. Since from Lemma 3,

$$
\Lambda_{q, \alpha}^{n} z^{k}=\frac{b_{k}\left(\alpha ; q^{2}\right)}{b_{k-n}\left(\alpha ; q^{2}\right)} z^{k-n}, \quad k \geq n,
$$

we can write

$$
g(z)=\sum_{n=0}^{\infty} \frac{\Lambda_{q, \alpha}^{n} g(0)}{b_{n}\left(\alpha ; q^{2}\right)} z^{n} .
$$

Using (4) and (6), we get

$$
\langle f, g\rangle_{\mathcal{F}_{q, \alpha}}=\sum_{n=0}^{\infty} a_{n} \overline{\Lambda_{q, \alpha}^{n} g(0)}=\sum_{n=0}^{\infty} a_{n} \Lambda_{q, \alpha}^{n} \tilde{g}(0) .
$$

Thus

$$
\langle f, g\rangle_{\mathcal{F}_{q, \alpha}}=f\left(\Lambda_{q, \alpha}\right) \tilde{g}(0),
$$

which gives the desired result.

The following theorem proves that $\mathcal{F}_{q, \alpha}$ is a reproducing kernel space.

Theorem 1. The function $\mathcal{K}_{q, \alpha}$ given for

$$
\begin{aligned}
& w, z \in D\left(0, \frac{1}{1-q}\right), \text { by } \\
& \mathcal{K}_{q, \alpha}(w, z)=E_{\alpha}\left(\bar{w} z ; q^{2}\right),
\end{aligned}
$$

is a reproducing kernel for the $q$-Fock space $\mathcal{F}_{q, \alpha}$, that is:

1) For all $w \in D\left(0, \frac{1}{1-q}\right)$, the function $z \rightarrow \mathcal{K}_{q, \alpha}(w, z)$ belongs to $\mathcal{F}_{q, \alpha}$.

2) For all $w \in D\left(0, \frac{1}{1-q}\right)$ and $f \in \mathcal{F}_{q, \alpha}$, we have

$$
\left\langle f, \mathcal{K}_{q, \alpha}(w, .)\right\rangle_{\mathcal{F}_{q, \alpha}}=f(w) .
$$

Proof. 1) Since

$$
\begin{aligned}
& \mathcal{K}_{q, \alpha}(w, z)=\sum_{n=0}^{\infty} \frac{\bar{w}^{n}}{b_{n}\left(\alpha ; q^{2}\right)} z^{n} ; \\
& z, w \in D\left(0, \frac{1}{1-q}\right),
\end{aligned}
$$

then from (3), we deduce that

$$
\left\|\mathcal{K}_{q, \alpha}(w, .)\right\|_{\mathcal{F}_{q, \alpha}}^{2}=\sum_{n=0}^{\infty} \frac{|w|^{2 n}}{b_{n}\left(\alpha ; q^{2}\right)}=E_{\alpha}\left(|w|^{2} ; q^{2}\right)<\infty,
$$

which proves 1$)$.

2) If $f(z)=\sum_{n=0}^{\infty} a_{n} z^{n} \in \mathcal{F}_{q, \alpha}$, from (4) and (7), we deduce

$$
\left\langle f, \mathcal{K}_{q, \alpha}(w, .)\right\rangle_{\mathcal{F}_{q, \alpha}}=\sum_{n=0}^{\infty} a_{n} w^{n}=f(w), \quad w \in D\left(0, \frac{1}{1-q}\right) .
$$

This completes the proof of the theorem.

Remark 2. From Theorem 12 ), for $f \in \mathcal{F}_{q, \alpha}$ and $w \in D\left(0, \frac{1}{1-q}\right)$, we have

$$
\begin{aligned}
|f(w)| & \leq\left\|\mathcal{K}_{q, \alpha}(w, .)\right\|_{\mathcal{F}_{q, \alpha}}\|f\|_{\mathcal{F}_{q, \alpha}} \\
& =\left[E_{\alpha}\left(|w|^{2} ; q^{2}\right)\right]^{1 / 2}\|f\|_{\mathcal{F}_{q, \alpha}} .
\end{aligned}
$$

Proposition 2. The space $\mathcal{F}_{q, \alpha}$ equipped with the inner product $\langle.,\rangle_{\mathcal{F}_{q, \alpha}}$ is an Hilbert space; and the set $\left\{\xi_{n}\left(. ; q^{2}\right)\right\}_{n \in \mathbb{N}}$ given by

$$
\xi_{n}\left(z ; q^{2}\right)=\frac{z^{n}}{\sqrt{b_{n}\left(\alpha ; q^{2}\right)}}, \quad z \in D\left(0, \frac{1}{1-q}\right),
$$

forms an Hilbert basis for the space $\mathcal{F}_{q, \alpha}$.

Proof. Let $\left\{f_{n}\right\}_{n \in \mathbb{N}}$ be a Cauchy sequence in $\mathcal{F}_{q, \alpha}$. We put

$$
f=\lim _{n \rightarrow \infty} f_{n}, \text { in } \mathcal{F}_{q, \alpha} .
$$

From (8), we have

$$
\left|f_{n+p}(w)-f_{n}(w)\right| \leq\left[E_{\alpha}\left(|w|^{2} ; q^{2}\right)\right]^{1 / 2}\left\|f_{n+p}-f_{n}\right\|_{\mathcal{F}_{q, \alpha}} .
$$

This inequality shows that the sequence $\left\{f_{n}\right\}_{n \in \mathbb{N}}$ is pointwise convergent to $f$. Since the function $w \rightarrow\left[E_{\alpha}\left(|w|^{2} ; q^{2}\right)\right]^{1 / 2}$ is continuous on $D\left(0, \frac{1}{1-q}\right)$, then $\left\{f_{n}\right\}_{n \in \mathbb{N}}$ converges to $f$ uniformly on all compact set of $D\left(0, \frac{1}{1-q}\right)$. Consequently, $f$ is an entire function on $D\left(0, \frac{1}{1-q}\right)$, then $f$ belongs to the space $\mathcal{F}_{q, \alpha}$.

On the other hand, from the relation (4), we get

$$
\left\langle\xi_{n}\left(. ; q^{2}\right), \xi_{m}\left(. ; q^{2}\right)\right\rangle_{\mathcal{F}_{q, \alpha}}=\delta_{n, m},
$$

where $\delta_{n, m}$ is the Kronecker symbol.

This shows that the family $\left\{\xi_{n}\left(. ; q^{2}\right)\right\}_{n \in \mathbb{N}}$ is an orthonormal set in $\mathcal{F}_{q, \alpha}$. 
Let $f(z)=\sum_{n=0}^{\infty} a_{n} z^{n}$ be an element of $\mathcal{F}_{q, \alpha}$ such that

$$
\left\langle f, \xi_{n}\left(. ; q^{2}\right)\right\rangle_{\mathcal{F}_{q, \alpha}}=0, \quad \forall n \in \mathbb{N} .
$$

From the relation (4), we deduce that

$$
a_{n}=0, \quad \forall n \in \mathbb{N} .
$$

This completes the proof.

Remark 3. 1) The set $\left\{E_{\alpha}\left(\bar{w} ; q^{2}\right), w \in D\left(0, \frac{1}{1-q}\right)\right\}$ is dense in $\mathcal{F}_{q, \alpha}$.

2) For all $z, w \in D\left(0, \frac{1}{1-q}\right)$, we have

$$
E_{\alpha}\left(w \bar{z} ; q^{2}\right)=\left\langle E_{\alpha}\left(\bar{z} ; q^{2}\right), E_{\alpha}\left(\bar{w} \cdot ; q^{2}\right)\right\rangle_{\mathcal{F}_{q, \alpha}} .
$$

\section{Operators on the Fock Spaces $\mathcal{F}_{q, \alpha}$}

On $\mathcal{F}_{q, \alpha}$, we consider the multiplication operators $Q$ and $N_{q}$ given by

$$
\begin{gathered}
Q f(z):=z f(z), \\
N_{q} f(z):=z D_{q} f(z)=\frac{f(z)-f(q z)}{1-q} .
\end{gathered}
$$

We denote also by $\Lambda_{q, \alpha}$ the $q$-Dunkl operator defined for entire functions on $D\left(0, \frac{1}{1-q}\right)$.

We write

$$
\left[\Lambda_{q, \alpha}, Q\right]=\Lambda_{q, \alpha} Q-Q \Lambda_{q, \alpha} .
$$

Then by straightforward calculation we obtain.

Lemma 4. $\left[\Lambda_{q, \alpha}, Q\right]=B_{q}+W_{q, \alpha}$, where

$$
\begin{gathered}
B_{q} f(z):=f(q z), \\
W_{q, \alpha}:=\frac{[2 \alpha+1]_{q}}{2}\left[(q-1) B_{q}+(q+1) B_{-q}\right] .
\end{gathered}
$$

Remark 4. The Lemma 4 is the analogous commutation rule of [3]. When $q \rightarrow 1^{-}$, then $\left[\Lambda_{q, \alpha}, Q\right]$ tends to $I+(2 \alpha+1) B$, where $I$ is the identity operator and $B$ is the parity operator given by $B f(z):=f(-z)$.

Lemma 5. If $f \in \mathcal{F}_{q, \alpha}$ then $B_{q} f, N_{q} f$ and $W_{q, \alpha} f$ belong to $\mathcal{F}_{q, \alpha}$, and

1) $\left\|B_{q} f\right\|_{\mathcal{F}_{q, \alpha}}=\left\|B_{-q} f\right\|_{\mathcal{F}_{q, \alpha}} \leq\|f\|_{\mathcal{F}_{q, \alpha}}$,

2) $\left\|N_{q} f\right\|_{\mathcal{F}_{q, \alpha}} \leq \frac{1}{1-q}\|f\|_{\mathcal{F}_{q, \alpha}}$,

3) $\left\|W_{q, \alpha} f\right\|_{\mathcal{F}_{q, \alpha}} \leq[2 \alpha+1]_{q}\|f\|_{\mathcal{F}_{q, \alpha}}$.

Proof. Let $f(z)=\sum_{n=0}^{\infty} a_{n} z^{n} \in \mathcal{F}_{q, \alpha}$, then

$$
\begin{gathered}
B_{q} f(z)=f(q z)=\sum_{n=0}^{\infty} a_{n} q^{n} z^{n}, \\
N_{q} f(z)=\frac{f(z)-f(q z)}{1-q}=\sum_{n=0}^{\infty} a_{n}[n]_{q} z^{n},
\end{gathered}
$$

and from (3), we obtain

$$
\begin{aligned}
\left\|B_{q} f\right\|_{\mathcal{F}_{q, \alpha}}^{2} & =\sum_{n=0}^{\infty}\left|a_{n}\right|^{2} q^{2 n} b_{n}\left(\alpha ; q^{2}\right) \\
& \leq \sum_{n=0}^{\infty}\left|a_{n}\right|^{2} b_{n}\left(\alpha ; q^{2}\right)=\|f\|_{\mathcal{F}_{q, \alpha}}^{2},
\end{aligned}
$$

and

$$
\left\|N_{q} f\right\|_{\mathcal{F}_{q, \alpha}}^{2}=\sum_{n=0}^{\infty}\left|a_{n}\right|^{2}\left([n]_{q}\right)^{2} b_{n}\left(\alpha ; q^{2}\right) .
$$

Using the fact that $[n]_{q} \leq \frac{1}{1-q}$, we deduce

$$
\left\|N_{q} f\right\|_{\mathcal{F}_{q, \alpha}}^{2} \leq \frac{1}{(1-q)^{2}} \sum_{n=0}^{\infty}\left|a_{n}\right|^{2} b_{n}\left(\alpha ; q^{2}\right)=\frac{1}{(1-q)^{2}}\|f\|_{\mathcal{F}_{q, \alpha}}^{2} .
$$

On the other hand from 1) we deduce that

$$
\begin{aligned}
& \left\|W_{q, \alpha} f\right\|_{\mathcal{F}_{q, \alpha}} \\
& \leq \frac{[2 \alpha+1]_{q}}{2}\left[(1-q)\left\|B_{q} f\right\|_{\mathcal{F}_{q, \alpha}}+(q+1)\left\|B_{-q} f\right\|_{\mathcal{F}_{q, \alpha}}\right] \\
& \leq[2 \alpha+1]_{q}\|f\|_{\mathcal{F}_{q, \alpha}},
\end{aligned}
$$

which completes the proof of the Lemma.

We now study the continuous property of the operators $\Lambda_{q, \alpha}$ and $Q$ on $\mathcal{F}_{q, \alpha}$.

Theorem 2. If $f \in \mathcal{F}_{q, \alpha}$ then $\Lambda_{q, \alpha} f$ and $Q f$ belong to $\mathcal{F}_{q, \alpha}$, and we have
1) $\left\|\Lambda_{q, \alpha} f\right\|_{\mathcal{F}_{q, \alpha}} \leq C_{q, \alpha}\|f\|_{\mathcal{F}_{q, \alpha}}$,
2) $\|Q f\|_{\mathcal{F}_{q, \alpha}} \leq C_{q, \alpha}\|f\|_{\mathcal{F}_{q, \alpha}}$, where

$$
C_{q, \alpha}:=\left([2 \alpha+1]_{q}+\frac{1}{1-q}\right)^{1 / 2} .
$$

Proof. Let $f(z)=\sum_{n=0}^{\infty} a_{n} z^{n} \in \mathcal{F}_{q, \alpha}$.

1) From Lemma 3 ,

$$
\begin{aligned}
\Lambda_{q, \alpha} f(z) & =\sum_{n=1}^{\infty} a_{n} \frac{b_{n}\left(\alpha ; q^{2}\right)}{b_{n-1}\left(\alpha ; q^{2}\right)} z^{n-1} \\
& =\sum_{n=0}^{\infty} a_{n+1} \frac{b_{n+1}\left(\alpha ; q^{2}\right)}{b_{n}\left(\alpha ; q^{2}\right)} z^{n} .
\end{aligned}
$$

Then from (9), we get

$$
\left\|\Lambda_{q, \alpha} f\right\|_{\mathcal{F}_{q, \alpha}}^{2}=\sum_{n=0}^{\infty}\left|a_{n+1}\right|^{2} \frac{b_{n+1}\left(\alpha ; q^{2}\right)}{b_{n}\left(\alpha ; q^{2}\right)} b_{n+1}\left(\alpha ; q^{2}\right) .
$$

Using Lemma 2 1), we obtain 


$$
\begin{aligned}
& \left\|\Lambda_{q, \alpha} f\right\|_{\mathcal{F}_{q, \alpha}}^{2} \\
& =\sum_{n=0}^{\infty}\left|a_{n}\right|^{2}\left[[n]_{q}+q^{n}[2 \alpha+1]_{q}\left(\frac{1-(-1)^{n}}{2}\right)\right] b_{n}\left(\alpha ; q^{2}\right) .
\end{aligned}
$$

Using the fact that $[n]_{q} \leq \frac{1}{1-q}$, we obtain

$$
\begin{aligned}
\left\|\Lambda_{q, \alpha} f\right\|_{\mathcal{F}_{q, \alpha}} & \leq C_{q, \alpha}\left[\sum_{n=0}^{\infty}\left|a_{n}\right|^{2} b_{n}\left(\alpha ; q^{2}\right)\right]^{1 / 2} \\
& =C_{q, \alpha}\|f\|_{\mathcal{F}_{q, \alpha}} .
\end{aligned}
$$

2) On the other hand, since

$$
Q f(z)=\sum_{n=1}^{\infty} a_{n-1} z^{n},
$$

then

$$
\|Q f\|_{\mathcal{F}_{q, \alpha}}^{2}=\sum_{n=1}^{\infty}\left|a_{n-1}\right|^{2} b_{n}\left(\alpha ; q^{2}\right)=\sum_{n=0}^{\infty}\left|a_{n}\right|^{2} b_{n+1}\left(\alpha ; q^{2}\right) .
$$

By Lemma 2 1), we deduce

$\|Q f\|_{\mathcal{F}_{q, \alpha}}^{2}$

$=\sum_{n=0}^{\infty}\left|a_{n}\right|^{2}\left[[n+1]_{q}+q^{n+1}[2 \alpha+1]_{q}\left(\frac{1+(-1)^{n}}{2}\right)\right] b_{n}\left(\alpha ; q^{2}\right)$.

Using the fact that $[n+1]_{q} \leq \frac{1}{1-q}$, we obtain

$$
\|Q f\|_{\mathcal{F}_{q, \alpha}} \leq C_{q, \alpha}\|f\|_{\mathcal{F}_{q, \alpha}} .
$$

We deduce also the following norm equalities.

Theorem 3. If $f \in \mathcal{F}_{q, \alpha}$ then

1) $\left\|\Lambda_{q, \alpha} f\right\|_{\mathcal{F}_{q, \alpha}}^{2}=\left\langle f, N_{q} f\right\rangle_{\mathcal{F}_{q, \alpha}}$

$$
+\frac{[2 \alpha+1]_{q}}{2}\left\langle f,\left(B_{q}-B_{-q}\right) f\right\rangle_{\mathcal{F}_{q, \alpha}},
$$

2) $\|Q f\|_{\mathcal{F}_{q, \alpha}}^{2}=\left\langle f, N_{q} f\right\rangle_{\mathcal{F}_{q, \alpha}}+\left\|B_{\sqrt{q}} f\right\|_{\mathcal{F}_{q, \alpha}}^{2}$

$$
+\frac{q[2 \alpha+1]_{q}}{2}\left\langle f,\left(B_{q}+B_{-q}\right) f\right\rangle_{\mathcal{F}_{q, \alpha}}
$$

3) $\|Q f\|_{\mathcal{F}_{q, \alpha}}^{2}=\left\|\Lambda_{q, \alpha} f\right\|_{\mathcal{F}_{q, \alpha}}^{2}+\left\|B_{\sqrt{q}} f\right\|_{\mathcal{F}_{q, \alpha}}^{2}+\left\langle f, W_{q, \alpha} f\right\rangle_{\mathcal{F}_{q, \alpha}}$.

Proof. Let $f(z)=\sum_{n=0}^{\infty} a_{n} z^{n} \in \mathcal{F}_{q, \alpha}$.

1) From (10), we get

$$
\begin{aligned}
& \left\|\Lambda_{q, \alpha} f\right\|_{\mathcal{F}_{q, \alpha}}^{2} \\
& =\sum_{n=0}^{\infty}\left|a_{n}\right|^{2}\left[[n]_{q}+q^{n}[2 \alpha+1]_{q}\left(\frac{1-(-1)^{n}}{2}\right)\right] b_{n}\left(\alpha ; q^{2}\right) \\
& =\left\langle f, N_{q} f\right\rangle_{\mathcal{F}_{q, \alpha}}+\frac{[2 \alpha+1]_{q}}{2}\left\langle f,\left(B_{q}-B_{-q}\right) f\right\rangle_{\mathcal{F}_{q, \alpha}} .
\end{aligned}
$$

2) On the other hand, by (12) and using the fact that $[n+1]_{q}=[n]_{q}+q^{n}$, we obtain

$$
\begin{aligned}
\|Q f\|_{\mathcal{F}_{q, \alpha}}^{2}= & \sum_{n=0}^{\infty}\left|a_{n}\right|^{2}[n]_{q} b_{n}\left(\alpha ; q^{2}\right)+\sum_{n=0}^{\infty}\left|a_{n}\right|^{2} q^{n} b_{n}\left(\alpha ; q^{2}\right) \\
& +\frac{q[2 \alpha+1]_{q}}{2} \sum_{n=0}^{\infty}\left|a_{n}\right|^{2} q^{n}\left(1+(-1)^{n}\right) b_{n}\left(\alpha ; q^{2}\right) \\
= & \left\langle f, N_{q} f\right\rangle_{\mathcal{F}_{q, \alpha}}+\|\left. B_{\sqrt{q}} f\right|_{\mathcal{F}_{q, \alpha}} ^{2} \\
& +\frac{q[2 \alpha+1]_{q}}{2}\left\langle f,\left(B_{q}+B_{-q}\right) f\right\rangle_{\mathcal{F}_{q, \alpha}} .
\end{aligned}
$$

3) Follows directly from 1) and 2).

Proposition 3. The operators $Q$ and $\Lambda_{q, \alpha}$ are adjoint-operators on $\mathcal{F}_{q, \alpha}$; and for all $f, g \in \mathcal{F}_{q, \alpha}$, we have

$$
\langle Q f, g\rangle_{\mathcal{F}_{q, \alpha}}=\left\langle f, \Lambda_{q, \alpha} g\right\rangle_{\mathcal{F}_{q, \alpha}} .
$$

Proof. Consider $f(z)=\sum_{n=0}^{\infty} a_{n} z^{n}$ and $g(z)=\sum_{n=0}^{\infty} c_{n} z^{n}$ in $\mathcal{F}_{q, \alpha}$. From (9) and (11),

$$
\Lambda_{q, \alpha} g(z)=\sum_{n=0}^{\infty} c_{n+1} \frac{b_{n+1}\left(\alpha ; q^{2}\right)}{b_{n}\left(\alpha ; q^{2}\right)} z^{n},
$$

and

$$
Q f(z)=\sum_{n=1}^{\infty} a_{n-1} z^{n} .
$$

Thus from (4), we get

$$
\begin{aligned}
\langle Q f, g\rangle_{\mathcal{F}_{q, \alpha}} & =\sum_{n=1}^{\infty} a_{n-1} \overline{c_{n}} b_{n}\left(\alpha ; q^{2}\right) \\
& =\sum_{n=0}^{\infty} a_{n} \overline{c_{n+1}} b_{n+1}\left(\alpha ; q^{2}\right)=\left\langle f, \Lambda_{q, \alpha} g\right\rangle_{\mathcal{F}_{q, \alpha}},
\end{aligned}
$$

which gives the result.

In the next part of this section we study a generalized translation and multiplication operators on $\mathcal{F}_{q, \alpha}$. We begin by the following definition.

Definition 2. For $f \in \mathcal{F}_{q, \alpha}$ and $w, z \in D\left(0, \frac{1}{1-q}\right)$, we define:

- The $q$-translation operators on $\mathcal{F}_{q, \alpha}$, by 


$$
T_{z} f(w):=E_{\alpha}\left(z \Lambda_{q, \alpha} ; q^{2}\right) f(w)=\sum_{n=0}^{\infty} \frac{\Lambda_{q, \alpha}^{n} f(w)}{b_{n}\left(\alpha ; q^{2}\right)} z^{n} .
$$

- The generalized multiplication operators on $\mathcal{F}_{q, \alpha}$, by

$$
M_{z} f(w):=E_{\alpha}\left(z Q ; q^{2}\right) f(w)=\sum_{n=0}^{\infty} \frac{Q^{n} f(w)}{b_{n}\left(\alpha ; q^{2}\right)} z^{n} .
$$

For $w, z \in D\left(0, \frac{1}{1-q}\right)$, the function $E_{\alpha}\left(. ; q^{2}\right)$ satisfies the following product formulas:

$$
\begin{gathered}
T_{z} E_{\alpha}\left(. ; q^{2}\right)(w)=E_{\alpha}\left(z ; q^{2}\right) E_{\alpha}\left(w ; q^{2}\right), \\
M_{z} E_{\alpha}\left(. ; q^{2}\right)(w)=E_{\alpha}\left(w z ; q^{2}\right) E_{\alpha}\left(w ; q^{2}\right) .
\end{gathered}
$$

Remark 5. If $q \rightarrow 1^{-}$in (13), we obtain the generalized translation operators given in ([9], page 102).

Proposition 4. Let $f(z)=\sum_{n=0}^{\infty} a_{n} z^{n} \in \mathcal{F}_{q, \alpha} \quad$ and $z, w \in D\left(0, \frac{1}{1-q}\right)$. Then

1) $T_{z} f(w)=\sum_{n=0}^{\infty} a_{n} \sum_{k=0}^{n} \frac{b_{n}\left(\alpha ; q^{2}\right)}{b_{k}\left(\alpha ; q^{2}\right) b_{n-k}\left(\alpha ; q^{2}\right)} w^{n-k} z^{k}$, with

$$
\begin{aligned}
& \frac{b_{n}\left(\alpha ; q^{2}\right)}{b_{k}\left(\alpha ; q^{2}\right) b_{n-k}\left(\alpha ; q^{2}\right)} \\
& =\frac{c\left(n, k ; q^{2}\right) \Gamma_{q^{2}}(\alpha+1) \Gamma_{q^{2}}\left(\left[\frac{n+1}{2}\right]+\alpha+1\right)}{\Gamma_{q^{2}}\left(\left[\frac{k+1}{2}\right]+\alpha+1\right) \Gamma_{q^{2}}\left(\left[\frac{n-k+1}{2}\right]+\alpha+1\right)},
\end{aligned}
$$

where

$$
c\left(n, k ; q^{2}\right)=\frac{\Gamma_{q^{2}}([n / 2]+1)}{\Gamma_{q^{2}}([k / 2]+1) \Gamma_{q^{2}}\left(\left[\frac{n-k}{2}\right]+1\right)} .
$$

2) $M_{z} f(w)=\sum_{n=0}^{\infty}\left[\sum_{k=0}^{n} \frac{a_{n-k}}{b_{k}\left(\alpha ; q^{2}\right)} z^{k}\right] w^{n}$.

Proof. Let $f(z)=\sum_{n=0}^{\infty} a_{n} z^{n} \in \mathcal{F}_{q, \alpha}$.

1) From (13), we have

$$
T_{z} f(w)=\sum_{n=0}^{\infty} \frac{\Lambda_{q, \alpha}^{n} f(w)}{b_{n}\left(\alpha ; q^{2}\right)} z^{n} ; \quad w, z \in D\left(0, \frac{1}{1-q}\right) .
$$

But from (5), we have

$$
\Lambda_{q, \alpha}^{n} f(w)=\sum_{k=n}^{\infty} a_{k} \frac{b_{k}\left(\alpha ; q^{2}\right)}{b_{k-n}\left(\alpha ; q^{2}\right)} w^{k-n} .
$$

Thus we obtain

$$
T_{z} f(w)=\sum_{n=0}^{\infty} a_{n} \sum_{k=0}^{n} \frac{b_{n}\left(\alpha ; q^{2}\right)}{b_{k}\left(\alpha ; q^{2}\right) b_{n-k}\left(\alpha ; q^{2}\right)} w^{n-k} z^{k} .
$$

On the other hand from Lemma 23 ), we get

$$
\begin{aligned}
& \frac{b_{n}\left(\alpha ; q^{2}\right)}{b_{k}\left(\alpha ; q^{2}\right) b_{n-k}\left(\alpha ; q^{2}\right)} \\
& =\frac{c\left(n, k ; q^{2}\right) \Gamma_{q^{2}}(\alpha+1) \Gamma_{q^{2}}\left(\left[\frac{n+1}{2}\right]+\alpha+1\right)}{\Gamma_{q^{2}}\left(\left[\frac{k+1}{2}\right]+\alpha+1\right) \Gamma_{q^{2}}\left(\left[\frac{n-k+1}{2}\right]+\alpha+1\right)},
\end{aligned}
$$

which gives the 1 ).

2) From (14), we have

$$
M_{z} f(w)=\sum_{n=0}^{\infty} \frac{Q^{n} f(w)}{b_{n}\left(\alpha ; q^{2}\right)} z^{n} ; \quad w, z \in D\left(0, \frac{1}{1-q}\right) .
$$

But from (11), we have

$$
Q^{n} f(w)=\sum_{k=n}^{\infty} a_{k-n} w^{k} .
$$

Thus we obtain

$$
M_{z} f(w)=\sum_{n=0}^{\infty}\left[\sum_{k=0}^{n} \frac{a_{n-k}}{b_{k}\left(\alpha ; q^{2}\right)} z^{k}\right] w^{n} .
$$

According to Theorem 2 we study the continuous property of the operators $T_{z}$ and $M_{z}$ on $\mathcal{F}_{q, \alpha}$.

Theorem 4. If $f \in \mathcal{F}_{q, \alpha}$ and $|z|<\frac{1}{(1-q)\left(2-q^{2 \alpha+1}\right)}$,

then $T_{z} f$ and $M_{z} f$ belong to $\mathcal{F}_{q, \alpha}$, and we have

1) $\left\|T_{z} f\right\|_{\mathcal{F}_{q, \alpha}} \leq E_{\alpha}\left(C_{q, \alpha}|z| ; q^{2}\right)\|f\|_{\mathcal{F}_{q, \alpha}}$,

2) $\left\|M_{z} f\right\|_{\mathcal{F}_{q, \alpha}} \leq E_{\alpha}\left(C_{q, \alpha}|z| ; q^{2}\right)\|f\|_{\mathcal{F}_{q, \alpha}}$,

where $C_{q, \alpha}$ are the constants of Theorem 2.

Proof. From (13) and Theorem 2 1), we deduce

$$
\begin{aligned}
\left\|T_{z} f\right\|_{\mathcal{F}_{q, \alpha}} & \leq \sum_{n=0}^{\infty}\left\|\Lambda_{q, \alpha}^{n} f\right\|_{\mathcal{F}_{q, \alpha}} \frac{|z|^{n}}{b_{n}\left(\alpha ; q^{2}\right)} \\
& \leq \sum_{n=0}^{\infty} \frac{\left(C_{q, \alpha}|z|\right)^{n}}{b_{n}\left(\alpha ; q^{2}\right)}\|f\|_{\mathcal{F}_{q, \alpha}} .
\end{aligned}
$$

Since $|z|<\frac{1}{(1-q)\left(2-q^{2 \alpha+1}\right)}$, then $C_{q, \alpha}|z|<\frac{1}{(1-q)^{2}}$, and therefore

$$
\left\|T_{z} f\right\|_{\mathcal{F}_{q, \alpha}} \leq E_{\alpha}\left(C_{q, \alpha}|z| ; q^{2}\right)\|f\|_{\mathcal{F}_{q, \alpha}},
$$

which gives the first inequality, and as in the same way 
we prove the second inequality of this theorem.

From Proposition 3 we deduce the following results.

Proposition 5. For all $f, g \in \mathcal{F}_{q, \alpha}$, we have

$$
\begin{aligned}
& \left\langle M_{z} f, g\right\rangle_{\mathcal{F}_{q, \alpha}}=\left\langle f, T_{\bar{z}} g\right\rangle_{\mathcal{F}_{q, \alpha}}, \\
& \left\langle T_{z} f, g\right\rangle_{\mathcal{F}_{q, \alpha}}=\left\langle f, M_{\bar{z}} g\right\rangle_{\mathcal{F}_{q, \alpha}} .
\end{aligned}
$$

We denote by $R_{z}$ the following operator defined on $\mathcal{F}_{q, \alpha}$ by

$$
\begin{aligned}
R_{z}:= & T_{\bar{z}} M_{z}-M_{\bar{z}} T_{z}=E_{\alpha}\left(\bar{z} \Lambda_{q, \alpha} ; q^{2}\right) E_{\alpha}\left(z Q ; q^{2}\right) \\
& -E_{\alpha}\left(\bar{z} Q ; q^{2}\right) E_{\alpha}\left(z \Lambda_{q, \alpha} ; q^{2}\right) .
\end{aligned}
$$

Then, we prove the following theorem.

Theorem 5. For all $f \in \mathcal{F}_{q, \alpha}$, we have

$$
\left\|M_{z} f\right\|_{\mathcal{F}_{q, \alpha}}^{2}=\left\|T_{z} f\right\|_{\mathcal{F}_{q, \alpha}}^{2}+\left\langle f, R_{z} f\right\rangle_{\mathcal{F}_{q, \alpha}} .
$$

Proof. From Proposition 5, we get

$$
\begin{aligned}
\left\|M_{z} f\right\|_{\mathcal{F}_{q, \alpha}}^{2} & =\left\langle f, T_{\bar{z}} M_{z} f\right\rangle_{\mathcal{F}_{q, \alpha}}=\left\langle f,\left(M_{\bar{z}} T_{z}+R_{z}\right) f\right\rangle_{\mathcal{F}_{q, \alpha}} \\
& =\left\|T_{z} f\right\|_{\mathcal{F}_{q, \alpha}}^{2}+\left\langle f, R_{z} f\right\rangle_{\mathcal{F}_{q, \alpha}} .
\end{aligned}
$$

\section{REFERENCES}

[1] C. A. Berger and L. A. Coburn, "Toeplitz Operators on the Segal-Bargmann Space," Transactions of the American Mathematical Society, Vol. 301, No. 2, 1987, pp. 813829. doi:10.1090/S0002-9947-1987-0882716-4
[2] V. Bargmann, “On a Hilbert Space of Analytic Functions and an Associated Integral Transform, Part I," Communications on Pure and Applied Mathematics, Vol. 14, No. 3, 1961, pp. 187-214. doi:10.1002/cpa.3160140303

[3] M. Sifi and F. Soltani, "Generalized Fock Spaces and Weyl Relations for the Dunkl Kernel on the Real Line," Journal of Mathematical Analysis and Applications, Vol. 270, No. 1, 2002, pp. 92-106. doi:10.1016/S0022-247X(02)00052-5

[4] F. M. Cholewinski, "Generalized Fock Spaces and Associated Operators,” SIAM Journal of Mathematical Analysis, Vol. 15, No. 1, 1984, pp. 177-202. doi:10.1137/0515015

[5] G. H. Jackson, “On a $q$-Definite Integrals,” The Quarterly Journal of Pure and Applied Mathematics, Vol. 41, No. 2, 1910, pp. 193-203.

[6] T. H. Koornwinder, "Special Functions and $q$-Commuting Variables,” Fields Institute Communications, Vol. 14, 1997, pp. 131-166.

[7] A. Fitouhi, M. M. Hamza and F. Bouzeffour, "The $q-j_{\alpha}$ Bessel Function,” Journal of Approximation Theory, Vol. 115, No. 1, 2002, pp. 144-166. doi:10.1006/jath.2001.3645

[8] F. Soltani, "Multiplication and Translation Operators on the Fock Spaces for the q-Modified Bessel Function,” The Advances in Pure Mathematics (APM), Vol. 1, No. 2, 2011, pp. 221-227. doi:10.4236/apm.2011.14039

[9] J. J. Betancor, M. Sifi and K. Trimèche, "Hypercyclic and Chaotic Convolution Operators Associated with the Dunkl Operator on $\mathbb{C}$," Acta Mathematica Hungarica, Vol. 106, No. 1-2, 2005, pp. 101-116. doi:10.1007/s10474-005-0009-1 